\title{
Future of Personalized Prescription in Psychiatry
}

\author{
Jose de Leon \\ University of Kentucky Mental Health Research Center at Eastern State Hospital, and University of Kentucky \\ Colleges of Medicine and Pharmacy, Lexington, Ky., USA; Psychiatry and Neurosciences Research Group \\ (CTS-549), Institute of Neurosciences, University of Granada, Granada, Spain
}

\begin{abstract}
Personalized prescription and pharmacogenomics are related concepts, but are not the same. The 'Introduction' describes the concept of pharmacogenomics, which can be included within personalized prescription, and the role of the Human Genome Project and the Food and Drug Administration in promoting advances in these concepts. In the author's comprehensive view of personalized prescription, clinicians need to consider genetic, environmental and personal variables when prescribing any medication. Known important genetic variables in specific drug responses can be explored by pharmacogenetic tests. Environmental variables - such as co-medication, herb supplements, foods, beverages and smoking - may be much more important than genetic factors for some drugs. Personal factors such as age, gender or medical illnesses (renal or hepatic insufficiency) may be crucial personal variables in the response to some other drugs. The pharmacological knowledge needed to understand personalized prescription includes pharmacokinetics and pharmacodynamic actions, efficacy and safety, idiosyncratic and dose-related adverse drug reactions, prescriber's role and therapeutic window, and linear versus non-linear pharmacokinetics. The applications of these pharmacological concepts in psychiatry are briefly reviewed. Risperidone personalized prescription is provided as an example by describing personalized risperidone selection and personalized risperidone dosing. The future of pharmacogenomic tests and personalized prescription in psychiatry is briefly summarized.

Copyright $\odot 2010$ S. Karger AG, Basel
\end{abstract}

\section{Introduction}

While the theme of this book is pharmacogenomics in psychiatry, this particular chapter focuses on personalized prescription rather than on pharmacogenomics. Personalized prescription and pharmacogenomics are related concepts, but are not the same. One of the problems in medicine is that many of its concepts are nonclassical (or fuzzy) concepts with unclear but overlapping limits. This section re- 
views the concept of pharmacogenomics, the role of the Human Genome Project, the concepts of personalized medicine and personalized prescription, and the crucial role of the Food and Drug Administration (FDA) in promoting clinical applications. The article then describes the necessary pharmacological knowledge for understanding personalized prescription and its applications in psychiatry, with personalized prescription of risperidone as an example. Finally, the future of pharmacogenomic testing and personalized prescription in psychiatry is summarized.

\section{Pharmacogenomics}

Pirmohamed [1] defined pharmacogenomics as the study of all genes in the genome that may determine a drug response. Pharmacogenomics has gained major impetus from technological advances and the Human Genome Project.

\section{Human Genome Project}

The end of the 20th century brought new hopes of a revolution in medicine based on our advancing knowledge of the human genome. The Human Genome Project, officially completed in 2000 [2], was a crucial step but led to exaggerated hopes. In an example of this hyperbolic optimism, McKusick [3] published a commentary in JAMA comparing the Human Genome Project with the revolutionary influence of a 16th century text on anatomy by Vesalius which led to major developments in medicine, including further applications of the scientific method and the development of other basic sciences.

The Human Genome Project was possible due to rapid advances in genetic technologies that made possible the parallel testing of many single nucleotide polymorphisms (SNP) with progressively lowered costs. Currently, one can test millions of SNPs for less than USD 1,000 per sample, and the price is rapidly decreasing. The onset of these rapid technological advances led to a Science editorial comment in 1997 that defined 'personalized prescription' as 'tailoring drugs to a patient's genetic makeup' and predicted that personalized prescription would 'soon' reach clinical practice [4]. More exact estimates for the year in which generalized use of personalized prescription would begin included 2015 according to a 1999 Time magazine article [5] and 2020 according to a 2001 JAMA article [6].

Recent developments have proven how naïve it was to think that human genome mapping would change medicine by 2015 or 2020 . We do not yet know the function of approximately one third of human genes; other types of genetic variations such as deletions or duplications, the so-called copy number variations $(\mathrm{CNV})$, may have been neglected [7]. Unfortunately, many of the current platforms and systems used for genotyping mainly pay attention to SNPs and neglect CNVs or less common genetic variations such as microsatellite polymorphisms and translocations, inversions and substitutions, which may have some pharmacogenomic relevance [7]. Finally, the relevance of epigenetics to human pharmacogenetic response is not well understood 
[8], but it is important to know that in one animal model, drug tolerance was caused by epigenetic mechanisms [8].

Since the 'genomic' boom, technological advances have facilitated the development of a new wave of parallel testing of multiple physiological substrates and of new disciplines - including transcriptomics, proteomics and metabolomics [9]. These new technologies, sometimes included under the heading of biomarkers, provide hundreds or thousands of pieces of data on each patient, but produce two types of intrinsic problems: untested statistical analyses and complex interpretation of the results. Regarding statistical problems, the traditional statistical tests were developed to test one or a few hypotheses in dozens or hundreds of patients (many more individuals than tested hypotheses), not to test hundreds and thousands of hypotheses in samples that frequently include fewer individuals than hypotheses. Several new statistical methods are being developed to manage these large statistical databases. The author has collaborated in attempts to use two statistical methods derived from engineering statistics: analyzing genetic data using data mining [10] and a derivation of sensitive analysis and systems engineering [11]. Despite these preliminary attempts, he acknowledges that there are no validated methods; moreover, the statistical method used in genome-wide association studies with thousands of patients and published in the best scientific journals demonstrates very poor replicability.

Results from some of the new tests or biomarkers are hard to interpret since we know little about the normal variations of physiological substrates. In the case of metabolomics, we know little about the normal values of the hundreds of lipids that can be found in human blood. Even if we focus on pharmacogenomics and on the simplest genetic variations, SNPs, we have limited understanding of how to extrapolate to the clinical environment a statistical association between a specific SNP and response to drug X in a well-controlled study. The specific SNP may be associated with functional changes, may not be associated with functional changes and may be linked with other functional SNPs, or may be explained by a false-positive result. The relationships between SNPs and gene function appear to be fairly complicated in some of the well-studied genes. A well-studied pharmacogenetic gene, cytochrome P450 2D6 (CYP2D6), has more than 90 known genetic variations (including SNPs and CNVs) and more than 60 alleles [12]. The functional effects of some of the rarest CYP2D6 alleles are not known despite being relatively easy to study using a phenotyping test that requires giving a pill and measuring urine metabolites. The racial variations are relatively well understood, and it is thought that measuring approximately 20 alleles may provide a reasonable amount of information to clinicians about CYP2D6 phenotypes and function [13]. In most patients, these 20 alleles would establish whether or not the enzyme is present, and, if present, whether it is under- or overactive. Unfortunately, learning the functionality of these genetic variations (the phenotype-genotype association or correlation) has taken over 20 years to develop. Only a few SNPs have been studied in most genes of possible interest in psychiatric pharmacogenomics; in most of them, the functional meaning of these SNPs is not known. The con- 
ceptual and scientific difficulties in extrapolating from basic research to clinical applications, usually called translational research, are usually ignored in the literature and may be among the major obstacles for applying pharmacogenomics or personalized prescription in the clinical environment.

\section{Concepts of Personalized Medicine and Personalized Prescription}

The concepts of personalized or individualized medicine and prescription are not new in medical parlance. However, genetic advances have made discussing 'personalized medicine' and 'personalized prescription' in genetic terms fashionable. In fact, even lay journals use these concepts frequently, referring mainly to genetic differences between patients. In introducing the first issue of the newly created journal Personalized Medicine, Ruaño [14] reminded us that physicians have traditionally practiced personalized medicine in their attempts to decide the best treatment for each of their patients. However, physicians were not using the term 'personalized medicine'; the personalized approach traditionally used by physicians was probably based on subjective physician preferences and not on scientific knowledge. In fact, psychiatrists had used the term in a completely different way. In 1952, Osborn [15] titled his psychiatry textbook Psychiatry and Medicine: An Introduction to Personalized Medicine. The idea behind that title was that each patient is a unique individual with unique psychological mechanisms. For Osborn, the principle that allowed personalized medicine was not genetics, but psychoanalysis. Obviously, Osborn's opinion appears somewhat outmoded. On the other hand, the current exclusive focus of personalized medicine on genetics may be wrong.

This author [7] views personalized medicine as a very global concept that may include 'personalized surgery', 'personalized rehabilitation', 'personalized nutrition' and other types of personalized medical interventions and, more importantly for psychiatrists, 'personalized prescription'. Personalized prescription should include not only the use of new tests, which may or may not be pharmacogenetic tests, but also the consideration of all scientific information valid for prescribing medication [16]. Pharmacology is a mechanistic science; knowing the pharmacological principles behind drug response allows predictions to be made (table 1). For many drugs, genetic factors may be irrelevant in drug response or may be much less important than other non-genetic factors. Our pharmacological knowledge of each drug should determine what aspects are important in that drug's personalized prescription. In this comprehensive view of personalized prescription, clinicians need to consider genetic, environmental and personal variables when prescribing any medication [16]. Known important genetic variables in specific drug response can be explored using pharmacogenetic tests. Environmental variables such as co-medication, herb supplements, foods, beverages, and smoking may be much more important than genetic factors for some drugs. Personal factors such as age, gender or medical illnesses (renal or hepatic insufficiency) may be crucial personal variables in the response to some other drugs. 
Table 1. Pharmacological principles behind the author's view of personalized prescription

1 Mechanistic science: Pharmacology is a mechanistic science.

2 Pharmacokinetics and pharmacodynamics: Drug response is explained by the pharmacokinetic and pharmacodynamic actions of the drugs. Pharmacokinetic actions are usually a first step, occurring prior to pharmacodynamic actions. Pharmacodynamic actions in the brain may require a greater level of complexity than pharmacodynamic actions in the periphery, due to the complexity of moving drugs from the blood to the brain target.

3 Genetic, environmental and personal variables: Influence drug pharmacokinetics and pharmacodynamics.

4 Pharmacogenetic tests: Any attempt to use pharmacogenomic tests to help with personalized prescription also needs to take into account environmental and personal variables. For some drug responses, genetic variations may be irrelevant or have relatively little influence when compared with environmental and personal variables.

5 Other important pharmacological principles: A pharmacogenomic test or any other method to personalize prescription for a specific drug or group of drugs can only be developed by taking into account those pharmacological principles that are important in explaining how that drug works in the real world. Personalized medicine must consider (in addition to pharmacokinetics and pharmacodynamics):

5.1. Efficacy and safety (safety includes idiosyncratic versus dose-related adverse drug reactions). 5.2. Prescriber's role and therapeutic window (therapeutic window includes linear versus nonlinear pharmacokinetics).

This author has also hypothesized that personalized prescription can be expressed in two main ways: in the clinical environment as personalized selection of the drug, and as personalized dosing [7]. The author's definition of personalized prescription may be original, but his approach is not new since it is based on advances of pharmacological knowledge that are usually expressed in the drug prescribing information required by the FDA and in pharmacological textbooks.

\section{Crucial Role of the FDA in Promoting the Use of Personalized Prescription and Pharmacogenetic Testing}

The FDA has had a crucial role in promoting the use of pharmacological information to personalize prescriptions and in the introduction of pharmacogenetic tests in the clinical environment. A remarkable step was the terfenadine story. Terfenadine is a non-sedating antihistaminic that was approved by the FDA in the late 1980s with an average recommended dosage for average subjects after rigorous placebo-controlled clinical trials in healthy subjects not taking other medications. Then, a second experiment, naturalistic and not well-controlled, began when terfenadine was given to the general population which included many non-average subjects who were ill or taking other medications. In 1996, it was clear that more than 100 people had died in the USA in this naturalistic experiment. The 'average' doses were toxic and caused 
arrhythmias in subjects taking some co-medications inhibiting the cytochrome P450 (CYP) isoenzyme that metabolizes terfenadine. Had our knowledge of CYP 'science' been better, the lethal outcomes during naturalistic use would have been avoided. The deaths of more than 100 people taking terfenadine were not in vain. The FDA began progressively forcing companies to study drug-drug interactions (DDIs) and CYP metabolism. Thus, drug package inserts (currently called prescribing information) were required to include progressively more information on DDIs (environmental variables) and on peculiar situations such as renal or liver insufficiency (personal variables). After terfenadine, several drugs were withdrawn from the market due to similar cases of heart toxicity associated with DDIs, leading to drug prescribing information that increasingly focused on non-average subjects by including information on the effects of environmental and personal variables in drug response.

A further development in the FDA's approach was the inclusion of genetic information in the drug prescribing information. This was not well-received by pharmaceutical companies which had not embraced pharmacogenomic testing in clinical practice. In fact, an FDA official related [17] that when she met with drug industry representatives in 2001 to discuss the promise of personalized medicine: 'People stood up and said: We are terrified'. It is very easy to explain this terror. The pharmaceutical companies' current business model assumes drug approval on the basis of an average dosage recommendation for an average patient. Thus, the practice of excluding some patients from the drug using pharmacogenomic tests would narrow market niches. On the other hand, genotyping and treating some patients with alternative dosages would complicate prescribing information relative to competing drugs [18, 19]. If a drug is approved with pharmacogenomic testing as a requirement, the marketers of previously approved competing drugs would surely remind physicians that their drugs do not have such a requirement in their prescription package, but the new drug does.

The FDA has progressively set new recommendations to promote pharmacogenetics and personalized prescription. In 2005, the FDA provided guidance for the drug industry regarding pharmacogenetic data submission [20] that described a metabolic enzyme important for psychiatry, CYP2D6, as a 'valid biomarker' and introduced the idea of a voluntary data submission program. In 2008, the FDA [21] issued draft guidance for 'in vitro diagnostic multivariate index assays' (IVDMIAs). Pharmacogenomic, metabolomic and proteomic tests are IVDMIAs, and thus the FDA was indicating its intent to require IVDMIAs to meet pre-market and post-market device requirements under FDA regulations. Prior to that, the FDA had not been involved in regulating diagnostic tests.

In addition, the FDA took two major practical steps: in 2006 it approved the first pharmacogenomic test, the AmpliChip CYP 450 Test $[18,19]$, and in 2007 it began requiring clinicians to use a pharmacogenomic test before administering carbamazepine in a particular racial subgroup [22].

Roche Molecular Systems, Inc., developed the first pharmacogenomic test approved by the FDA, the AmpliChip CYP 450 Test. The microarray contains over 
Efficacy

Pharmacological mechanisms explaining drug response
Safety

Pharmacological mechanisms explaining ADRs

1 Dose-dependent ADRs

- Explained by an augmentation of the pharmacological action

2 Idiosyncratic ADRs

- Not predicted by pharmacological action

- Two main mechanisms are suggested: peculiar metabolic pathways leading to reactive metabolites and/or immunological response

Fig. 1. Efficacy and safety. $A D R s=$ Adverse drug reactions.

15,000 oligonucleotide probes allowing testing for 20 CYP2D6 alleles, 7 CYP2D6 duplications, and 3 cytochrome P450 2C19 (CYP2C19) alleles [18, 19]. CYP2D6 is particularly important in psychiatry since it metabolizes many antipsychotics and antidepressants. CYP2D6 poor metabolizers (PM) lack CYP2D6 in their bodies; their prevalence varies by race (highest among Caucasians, approximately $7 \%$, and lower in other races, 1-3\%). The psychiatric applications of CYP testing have been described in prior articles $[12,23,24]$.

The FDA required that HLA-B*1502 genotyping in Asians should be performed before prescribing carbamazepine to avoid the almost certain development of Stevens Johnson syndrome/toxic epidermal necrolysis in those with this marker [22].

In conclusion, in the view of the author: (1) pharmacogenomic tests are one type of personalized prescription test, and (2) personalized prescription should consider the influence of genetic, environmental and personal variables on each drug.

\section{Pharmacological Knowledge Needed for Personalized Prescription}

Table 1 describes drug response as being explained by pharmacokinetic and pharmacodynamic actions influenced by genetic, environmental and personal variables. Pharmacogenetic testing, and any other form of personalized prescription, must focus on other basic pharmacological principles besides pharmacokinetics and pharmacodynamics. This chapter cannot include a pharmacology textbook; therefore, tables and figures are used to remind the reader of crucial pharmacological concepts. Efficacy and safety are important concepts described in figure 1. Within safety, idiosyncratic versus dose-related adverse drug reactions (ADRs) must be distinguished [7]. Similarly, the prescriber's role and therapeutic window or index needs to be considered [16]. Pharmacology is very important in predicting drug response in drugs with a narrow therapeutic index. The prescriber's choice may be more relevant than pharmacological 


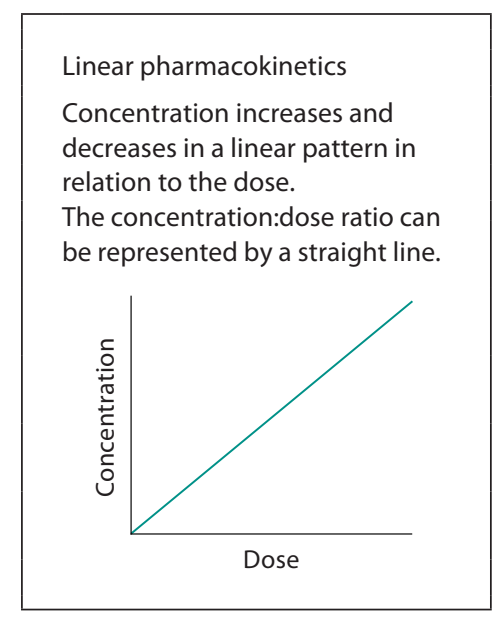

Non-linear pharmacokinetics

The concentration:dose ratio CANNOT be represented by a line making it more difficult to personalize dosing.

Several pharmacological mechanisms can explain this:

- Drugs that are auto-inhibitors of their own metabolism (e.g. paroxetine and fluoxetine)

- Drugs that are auto-inducers of their own metabolism (e.g. carbamazepine)

- Changes influenced by protein-binding and free concentration (e.g. valproate)

- Saturation kinetics: in high concentrations the half-life increases dramatically (e.g. phenytoin)

- Saturable absorption (e.g. gabapentin absorption decreases at higher doses)

Fig. 2. Comparing linear versus non-linear pharmacokinetics. Valproate concentration does not increase proportionally with the dose, but increases to a lesser extent due to saturable plasma protein binding. It is believed that the binding rate is $85-95 \%$ at low doses and $70 \%$ at high doses. Phenytoin has a narrow therapeutic window and follows non-linear pharmacokinetics, which is dosedependent and capacity-limited. This means that it is possible to attain excessive drug concentrations with modest dosage increases. When the drug reaches toxic levels phenytoin half-life may increase in a dramatic way due to the saturation of its metabolism. In fact, half-lives as long as $140 \mathrm{~h}$ have been described in intoxicated patients. Gabapentin appears to move from the intestine to the blood using a saturable L-amino acid transporter; gabapentin bioavailability is not doseproportional and decreases at higher doses.

Table 2. Comparing wide and narrow therapeutic window drugs

\begin{tabular}{lll}
\hline & Wide & Narrow \\
\hline $\begin{array}{lll}\text { Psychiatric drugs } & \text { most of the new drugs } & \text { most of the old drugs } \\
\text { Most relevant in dosing } & \text { prescriber's choice } & \text { pharmacology } \\
\text { relatively non-toxic } & \text { relatively toxic } \\
\text { Efficacy } & \text { low concentrations may be ineffective } & \text { varies for different individuals } \\
\text { Therapeutic drug monitoring } & \text { not well studied } & \text { used in clinical practice } \\
\text { Personalized dosing models } & \text { explain little of the drug response } & \text { explain more of the drug response } \\
\text { Pharmacogenomic tests } & \text { poor predictors of dosing } & \text { better predictors of dosing }\end{array}$ \\
\hline
\end{tabular}

principles in drugs with a wide therapeutic index. Table 2 elaborates on how the therapeutic window may influence personalized dosing. Another pharmacological concept that is important in dosing is linear versus non-linear pharmacokinetics (fig. 2). Pharmacokinetic type is fundamental in establishing personal dosing and in predicting the relationships between drug dose and drug blood concentration. It is easy to predict concentration using dose with drugs displaying linear kinetics, but more complicated with non-linear kinetics, which is present in some psychiatric drugs (fig. 2). 
By now it should be evident to the reader that it is impossible to begin thinking about how to apply personalized prescription in clinical practice without a thorough understanding of the pharmacokinetic and pharmacodynamic principles of psychiatric drugs. Table 3 includes a brief attempt to summarize the pharmacological mechanisms of the most important psychiatric drugs. Table 4 explains the pharmacological principles behind personalized drug selection, and provides psychiatric examples of personalized drug selection in clinical practice. Table 5 explains the pharmacological principles behind personalized dosing, and provides psychiatric examples of personalized drug selection in clinical practice. Personalized drug selection and dosing in psychiatry have been described in more detail in a prior article [7].

\section{Personalized Prescription of Risperidone as an Example}

The only way to completely understand what personalized prescription may mean in clinical practice is to provide a good example, such as risperidone. Risperidone prescription may need to take into account genetic, environmental and personal variations. In this article, it is not possible to extensively review the pharmacokinetics and pharmacodynamics of risperidone, which were reviewed in prior articles [25-28] and summarized in tables 6 and 7. Table 6 presents personalized risperidone selection. Basic pharmacological information relevant for risperidone selection is presented in the upper part of the table. Once a clinician has decided that an antipsychotic is needed, the lower part of table 6 describes which factors may be considered for or against risperidone selection. The information in the literature is very limited; multiple pragmatic randomized trials such as the Clinical Antipsychotic Trials of Intervention Effectiveness (CATIE) [29] are needed to correctly inform this decision. Due to the limited information available, and since clinicians frequently have drug preferences and dislikes, different clinicians would likely disagree on whether risperidone is a good first-choice antipsychotic for various patients. The existence of generic and long-acting formulations is a factor in favor of risperidone selection.

Table 7 presents personalized risperidone dosing. Basic pharmacological information relevant for risperidone dosing is presented in the upper part of the table. This table is based more on available scientific and pharmacological principles than the table focused on risperidone selection. As risperidone probably follows linear kinetics in all age groups [28], information on the effects of factors that change risperidone pharmacokinetics in adults may reasonably be extrapolated to children and geriatric patients. Three major factors are considered relevant when adapting risperidone dosing in patients with different personal characteristics: the presence of CYP3A inducers and/or CYP inhibitors, and CYP2D6 PM status (table 6). CYP2D6 PMs lack CYP2D6, the enzyme that has higher affinity for risperidone and may be the most 
Table 3. A brief summary of pharmacological mechanisms used to develop personalized prescription in psychiatry

1 Pharmacological principles behind the pharmacodynamics of efficacy are poorly understood and cannot easily be studied in vivo in patients.

1.1 Antipsychotics probably work as $\mathrm{D}_{2}$ blockers.

1.2 The majority of antidepressants are thought to be serotonin and/or noradrenaline reuptake inhibitors, but we are not sure whether this explains their antidepressant actions.

1.3 It is not known or well understood how antidepressants work in anxiety disorders.

1.4 Benzodiazepines probably act as allosteric modulators of the $G A B A_{A}$ receptors.

1.5 It is not known how the so-called mood stabilizers work.

2 Pharmacological principles behind the brain pharmacodynamics of safety are better understood.

2.1 Increases in appetite secondary to most antipsychotics and some antidepressants may be mainly mediated by $\mathrm{H}_{1}$ and $5-\mathrm{HT}_{2 \mathrm{C}}$ blockade.

2.2 Sedation secondary to antipsychotics and some antidepressants may be mainly mediated by $\mathrm{H}_{1}$, and muscarinic blockade.

2.3 Sedation secondary to benzodiazepines and some mood stabilizers may be mediated by GABAergic actions.

2.4 Extrapyramidal symptoms secondary to antipsychotics are mediated by $\mathrm{D}_{2}$ blockade.

2.5 Hyperprolactinemia secondary to antipsychotics is mediated by $\mathrm{D}_{2}$ blockade.

2.6 Cognitive impairment secondary to some antipsychotics and some antidepressants may be mediated by muscarinic blockade.

2.7 The serotonin syndrome secondary to the combinations of antidepressants, lithium and/or other drugs is believed to be explained by increased central and peripheral serotonin release.

3 Peripheral pharmacodynamic mechanisms are not always well studied.

3.1 Changes in heart rate and blood pressure secondary to some antidepressants and some antipsychotics may be mediated by the blockade of $\alpha$ - and $\beta$-adrenergic receptors and muscarinic receptors (central components are possible).

3.2 Constipation and urinary retention secondary to some antidepressants and antipsychotics are mainly mediated by muscarinic blockade.

3.3 Sexual ADRs and urinary incontinence secondary to some antipsychotics may be mediated by adrenergic blockade.

3.4 Mechanisms of peripheral glucose and lipid metabolism disturbed by antipsychotics are not well understood. Peripheral glucose and lipid metabolism actions of antidepressants and mood stabilizers are not well studied.

3.5 Platelet, gastrointestinal and sexual ADRs from some antidepressants are probably mediated by peripheral serotonergic changes.

3.6 Cardiac ion channel actions produced by some antidepressants and antipsychotics are not well studied. How these actions contribute to increased sudden deaths is not well understood.

3.7 Potentially lethal ADRs secondary to antipsychotics and mood stabilizers in skin, liver and hematological tissues are not understood and in some cases are possibly immunologically mediated.

4 Pharmacokinetic mechanisms are better understood but tend to be ignored by psychiatrists.

4.1 CYP.

- CYP2D6 is important for some antipsychotics, some antidepressants and activation of some opioids.

- CYP3A is important for some antipsychotics, some antidepressants, carbamazepine and some benzodiazepines.

- CYP2C19 is important for some antidepressants and diazepam.

- CYP1A2 is important for clozapine and olanzapine.

4.2 UGT. These poorly understood enzymes are important for lamotrigine, valproate, some opioids and secondary pathways for some antipsychotics and possibly some antidepressants.

4.3 Transporters are poorly understood.

- P-glycoprotein may be important at the intestine, liver and blood-brain barrier.

- Kidney transporters may be important for lithium and other drugs. 
Table 4. Pharmacological principles behind personalized drug selection and examples in psychiatry

1 Complexity: Personalizing drug selection is complicated since it requires considering multiple drugs.

2 Risk/benefit: The risk and benefits of each drug need to be considered.

3 Indication: The level of complexity is much higher when drugs from different classes can be selected (e.g. mania, which includes mood stabilizers and antipsychotics) versus only one class (e.g. a non-affective psychotic episode, which only includes antipsychotics).

4 Personal opinions: Personal opinions of physicians and patients on particular drugs and ADRs may be important in personalizing drug selection and are hard to predict using statistical models.

Examples of personalized drug selection in clinical practice

1 Contraindication of some drugs in some unusual subjects:

- Genetic variation. HLA-B*1502 genotyping in Asians: contraindicates carbamazepine.

- Environmental variation. Taking drugs that increase QTc: contraindicates ziprasidone.

- Personal variation. Pregnancy: contraindicates valproate.

2 Exclusion of some drugs in some patients due to some frequent ADRs not common to all drugs in one class:

- Genetic variations. Variants associated with TD risk: recommend against some antipsychotics.

- Environmental variations. Prescription of drugs associated with obesity: recommend against antipsychotics with greater risk of causing obesity.

- Personal variations. Elderly females are prone to develop TD: recommend against antipsychotics associated with greater TD risk.

3 Best drug for each patient:

This is an elusive goal beyond our current knowledge.

TD = Tardive dyskinesia.

${ }^{1}$ For more details, see de Leon [7].

important metabolic pathway for risperidone by hydroxylating it to 9-hydroxyrisperidone. PM patients can be identified by CYP2D6 genotyping [18, 19] or by measuring risperidone trough levels in steady state [23]. If the patient has a plasma risperidone/9-hydroxyrisperidone concentration ratio $>1$ and is not taking a CYP2D6 inhibitor (bupropion, fluoxetine or paroxetine) then he/she is likely to be a CYP2D6 PM. In the absence of these drugs, if the risperidone/9-hydroxyrisperidone ratio is 2 or 3, the patient is most definitively a CYP2D6 PM. Prior articles [23, 25-28] described this in detail. One of the articles described a pharmacokinetic model including genetic and environmental variables that allowed an exploration of their effects in dosing [26].

\section{Future of Pharmacogenomic Testing in Psychiatry}

The only FDA-required pharmacogenomic test in psychiatry is a test for one drug and for one racial group [22], and it only eliminates the risk of a relatively rare idiosyncratic ADR (HLA-B*1502 genotyping in Asians for carbamazepine). Clinicians have complained to the author that having one pharmacogenomic test for drug selection in one race is a miniscule advance. Unfortunately, this is the only pharmacogenomic test in the immediate future of psychiatry that has definitive support for its clinical indication. 
Table 5. Pharmacological principles behind personalized dosing and examples in psychiatry

1 Pharmacological principles behind dosing are much simpler than those behind drug selection.

2 Bottle-neck situations. One needs to remember that bottle-neck situations may apply at crucial points in drug response.

- $\quad$ Absorption disturbances by food ${ }^{1}$, other drugs $^{2}$, or even anatomical problems ${ }^{3}$ may decrease a drug's availability and render a drug ineffective.

- It is not well understood whether difficulties in crossing the BBB may be relevant in psychotropic drugs. Problems at the BBB have been explored as a possible mechanism for explaining lack of anticonvulsant response. Differences in BBB permeability may explain differences in toxicity between risperidone and its metabolite, 9-hydroxyrisperidone, marketed as paliperidone.

3 Dosing models combining genetic, environmental and personal variables may focus on:

3.1 Pharmacokinetic models: Models predicting blood drug concentration. Developing models for drugs following linear kinetics is much easier than for those drugs following non-linear kinetics (table 4). The clinical relevance of these models depends on how well blood concentration predicts drug response (efficacy and safety).

3.2 Clinical models: Predicting ADRs. They should incorporate pharmacokinetic and pharmacodynamic information. The ADR type is important:

3.2.1 Idiosyncratic ADRs: It makes no sense to try to develop dosing models since they are not dose related. Idiosyncratic ADRs may need certain doses (probably low) and after that the dose is irrelevant. Thus, it is better to deal with these ADRs by personalizing drug selection.

3.2.2 Dose-dependent ADRs: Dosing models may have better potential. However, it is important to consider whether tolerance develops or not.

3.2.2.1 ADRs not subject to tolerance: It may be easier to develop predictive dosing models. The narrower the therapeutic window, the more likely that the predictive model may work in the clinical environment.

3.2.2.2 ADRs subject to tolerance: High dosing may predict toxicity only in the initial doses. After some initial doses the patient may develop tolerance to ADRs. The predictive model for dosing needs to consider the duration of the treatment (initial versus maintenance).

4 The role of therapeutic window.

4.1 Wide therapeutic window drugs:

- Genetic variations: Being a UM may explain lack of efficacy.

- $\quad$ Environmental variations: Taking inducers may explain lack of efficacy.

4.2 Narrow therapeutic window drugs:

- Genetic variations:

Being a UM may explain lack of efficacy when the drug is metabolized.

Being a PM may explain lack of efficacy when the drug is activated.

Being a UM may explain ADRs when the drug is activated.

Being a PM may explain ADRs when the drug is metabolized.

- Environmental variations:

Taking inducers may explain lack of efficacy when the drug is metabolized.

Taking inhibitors may explain lack of efficacy when the drug is activated.

Taking inhibitors may explain ADRs when the drug is metabolized.

- Personal variations: Renal insufficiency may explain ADRs when the drug is excreted by the kidney.

Examples in clinical practice of personalized drug dosing 4

- $\quad$ Genetic variations: CYP2D6 PMs need half the usual dosages of TCAs.

- Environmental variations: Taking fluvoxamine requires lower clozapine dosing.

- Personal variations: Advanced age requires decreasing risperidone dosing.

$\mathrm{BBB}=$ Blood-brain barrier; $\mathrm{PM}=$ poor metabolizer; $\mathrm{UM}=$ ultrarapid metabolizer.

${ }^{1}$ Ziprasidone needs to be administered with food to increase its absorption.

${ }^{2}$ Carbamazepine suspension should not be administered with chlorpromazine suspension since they may lead to a precipitate and loss of absorption.

${ }^{3}$ Paliperidone capsules should be avoided in individuals with gastrointestinal narrowing which may hinder passage of a capsule through the gastrointestinal tract.

${ }^{4}$ For more details, see de Leon [7]. 
Table 6. Personalizing risperidone selection

Pharmacokinetic and pharmacodynamic profiles to consider

1 Important pharmacokinetic differences (which can be handled by dosing, see table 7)

- Risperidone versus non-dependent CYP2D6 antipsychotics (e.g. clozapine, olanzapine, paliperidone, quetiapine and ziprasidone): risk of overdosing in CYP2D6 PMs. This can be corrected by dosing.

- Clinically relevant DDI with CYP inhibitors (relatively similar to other antipsychotics except for ziprasidone and paliperidone, which may be relatively free of them). This can be corrected by dosing.

- Clinically relevant DDI with inducers (relatively similar to other antipsychotics except for ziprasidone, which may be relatively free of them). This can be corrected by dosing.

- Risperidone versus antipsychotics with limited renal excretion (e.g. clozapine, olanzapine, quetiapine and ziprasidone): risk of overdosing patients with renal insufficiency. This can be corrected by dosing.

2 Safety profile

- Increasing QTc: low risk (better than first-generation APs and ziprasidone).

- EPS: intermediate risk (better than first-generation APs and worse than other second-generation APs).

- Metabolic ADRs (obesity, hyperglycemia and hyperlipidemia): intermediate.

- Hyperprolactinemia: high risk (similar to first-generation APs and worse than other second generation APs).

- Sedation: low risk (better than clozapine, olanzapine, phenothiazines and quetiapine).

- Orthostatic hypotension: high risk (probably similar to clozapine, phenothiazines, quetiapine and ziprasidone).

- Sexourinary symptoms: rare (but probably higher than other second-generation APs).

- Gl symptoms: lower than the worst APs (aripiprazole and ziprasidone).

- Seizure: probably average risk (lower than the worst APs: chlorpromazine, clozapine, olanzapine and quetiapine).

- Liver toxicity: very low risk (lower than the worst APs: clozapine, olanzapine and phenothiazines).

- Antimuscarinic ADRs: no risk or very low risk (versus those APs with high risk: clozapine, olanzapine, phenothiazines and quetiapine). Remember that due to the potential for EPS, anticholinergic drugs may be needed when taking risperidone.

Personalizing risperidone selection within antipsychotics

1 Risperidone is contraindicated by:

- History of prior severe ADRs on risperidone (and possibly on paliperidone).

- Parkinson's disease and other neurodegenerative illnesses with parkinsonian symptoms. Use aripiprazole, clozapine or quetiapine.

- Current or past prolactin-sensitive cancer. If an AP is really needed, use an AP not associated with relevant prolactin elevations (aripiprazole is better or clozapine).

2 Risperidone may not be a good choice (other APs may be better):

- When orthostatic changes are a risk (serious cardiovascular disease or treatment with antihypertensives): Better choices may be aripiprazole, haloperidol, olanzapine or paliperidone.

- History of EPS in first-generation antipsychotics. Better choices are any of the other second-generation APs except paliperidone.

- Metabolic syndrome: risperidone has an intermediate position (worse than aripiprazole, haloperidol, molindone or ziprasidone; better than clozapine, olanzapine, phenothiazines and quetiapine).

3 Positive aspects of risperidone formulation:

- Generic is available (cheaper than other second-generation APs, and more expensive than first-generation APs).

- Long-acting is available (only others available in USA are fluphenazine, haloperidol and paliperidone).

APs $=$ Antipsychotics; EPS = extrapyramidal symptoms. 
Currently, CYP2D6 and CYP2C19 genotyping appear to have little future. Pharmaceutical companies are eliminating drugs metabolized by CYP2D6 from their pipeline $[18,19]$. First-generation antipsychotics tend to be CYP2D6 drugs and may be as efficacious as the new ones. As they are much cheaper, marketing the use of firstgeneration antipsychotics plus personalized tests, including CYP2D6 genotyping, may be the way to go [11]. Unfortunately, this idea is contrary to the current marketing strategies of the pharmaceutical companies which promote second-generation antipsychotics. Other psychiatric pharmacogenomic tests for clozapine efficacy, clozapine-induced agranulocytosis and antipsychotic-induced metabolic syndrome have been described in prior articles $[9,24]$.

The author believes that in the near future, in psychiatry, pharmacogenetic tests or other types of complex biomarkers have some potential in two areas [7]: (1) excluding the use of some drugs for some unusual patients (has major potential since neurology provided the first pharmacogenetic test for carbamazepine), and (2) personalizing drug dosing by using pharmacokinetic genes in narrow therapeutic window drugs (has some potential, but these drugs may be irrelevant for clinical practice unless the old antipsychotics are returned to use). There is dubious potential for: (1) selecting some drugs within a class due to ADR or efficacy profile, and (2) selecting dosing in a wide therapeutic window drug. The author does not see short-term potential in finding the best drug for each patient. This 'very sophisticated' level of personalized prescription is beyond our current knowledge and study methodologies [7].

\section{Future of Personalized Prescription in Psychiatry}

This author defines a new way of looking at personalized prescription, describing it as the use of genetic, environmental or personal information for selecting drugs and/ or prescribing dosages. With this broad definition, personalized prescription can be utilized without waiting for new developments in pharmacogenomic or other biomarker testing. Personalized prescription requires only that sophisticated clinicians understand that genetic, environmental or personal variables influence pharmacokinetic and pharmacodynamic response; the therapeutic window of the drug may also be important. Blood levels, currently called therapeutic drug monitoring, have been used by psychiatrists to personalize dosing for lithium, tricyclic antidepressants and some antipsychotics including clozapine. Unfortunately, all of these are old drugs rarely used by young prescribers in psychiatry. New drug marketing has convinced psychiatrists that they do not need to use these old drugs; thus, using therapeutic drug monitoring in psychiatry appears irrelevant. It also makes teaching this broad view of personalized prescription difficult. 


\section{Conclusions}

The 'Introduction' describes the concept of pharmacogenomics that can be included within personalized prescription and the role of the Human Genome Project and the FDA in promoting the advancement of these concepts. Personalized prescription and pharmacogenomics are related concepts, but are not the same. In the author's comprehensive view of personalized prescription, clinicians need to consider genetic, environmental and personal variables when prescribing any medication. The pharmacological knowledge needed to understand personalized prescription and its applications in psychiatry includes pharmacokinetic and pharmacodynamic actions, efficacy and safety, idiosyncratic and dose-related ADRs, prescriber's role and therapeutic window, and linear versus non-linear pharmacokinetics. Risperidone personalized prescription is provided as an example by describing personalized risperidone selection and personalized risperidone dosing. The future of pharmacogenomic tests and personalized prescription in psychiatry is briefly summarized.

\section{Acknowledgments}

The author thanks Lorraine Maw, MA, and Margaret T. Susce, RN, MLT, for editorial assistance.

\section{Disclosure and Conflict of Interest}

Since 10/1/2008, J.d.L. has been a co-investigator in an NIH Small Business Innovation Research Grant awarded to Genomas, Inc. He has received researcher-initiated grants from Eli Lilly (one ended in 2003 and the other, as co-investigator, ended in 2007) and from Roche Molecular Systems, Inc. (ended in 2007). He has been on the advisory board of Bristol-Myers Squibb (2003/04), AstraZeneca (2003) and Roche Molecular Systems, Inc. (2006). His lectures have been supported once by Sandoz (1997), twice by Lundbeck (1999 and 1999), twice by Pfizer (2001 and 2001), three times by Eli Lilly $(2003,2006$, and 2006), twice by Janssen (2000 and 2006), once by Bristol-Myers Squibb (2006), and eight times by Roche Molecular Systems, Inc. (twice in 2005 and six times in 2006).

\section{References}

1 Pirmohamed M: Pharmacogenetics and pharmacogenomics. J Clin Pharmacol 2001;52:345-347.

2 Golden F, Lemonick MD: The race is over. Time, 2000, pp 18-23.

3 McKusick VA: The anatomy of the human genome: a neo-vesalian basis for medicine in the 21st century. JAMA 2001;286:2289-2295.

4 New research horizons. Science 1997;278:2039.

5 Lertola J: Deciphering the code and what might come from it. Time, 1999, pp 68-69.
6 Collins FS, McKusick, VA: Implications of the human genome project for medical science. JAMA 2001;285:540-544.

7 de Leon J: The future (or lack of future) of personalized prescription in psychiatry. Pharmacol Res 2009;59:81-89.

8 Nebert DW, Zhang G, Vesell ES: From human genetics and genomics to pharmacogenetics and pharmacogenomics: past lessons, future directions. Drug Metab Rev 2008;40:187-224. 
$\checkmark$ de Leon J: Pharmacogenomics: the promise of personalized medicine for CNS disorders. Neuropsychopharmacology 2009;34:159-172.

10 Baca-Garcia E, Vaquero-Lorenzo C, Perez-Rodriguez MM, Gratacòs M, Bayés M, Santiago-Mozos R, Leiva-Murillo JM, de Prado-Cumplido M, ArtesRodriguez A, Ceverino A, Diaz-Sastre C, Fernandez-Navarro P, Costas J, Fernandez-Piqueras J, Diaz-Hernandez M, de Leon J, Baca-Baldomero E, Saiz-Ruiz J, Mann JJ, Parsey RV, Carracedo A, Estivill X, Oquendo MA: Nucleotide variation in central nervous system genes among male suicide attempters. Am J Med Genet B Neuropsychiatr Genet 2010;153B:208-213.

-11 Ruaño G, Goethe JW, Caley C, Woolley S, Holford TR, Kocherla M, Windemuth A, de Leon J: Physiogenomic comparison of weight profiles of olanzapine- and risperidone-treated patients. Mol Psychiatry 2007;12:474-482.

$\checkmark 12$ de Leon J, Susce MT, Johnson M, Hardin M, Maw L, Shao A, Allen AC, Chiafari FA, Hillman G, Nikoloff DM: DNA microarray technology in the clinical environment: the AmpliChip CYP 450 Test for CYP2D6 and CYP2C19 genotyping. CNS Spectr 2009;14:19-34.

13 Cai WM, Nikoloff DM, Pan RM, de Leon J, Fanti P, Fairchild M, Koch WH, Wedlund PJ: CYP2D6 genetic variations in healthy adults in psychiatric African-American subjects: implications for clinical practice and genetic testing. Pharmacogenom J 2006;6:343-350.

14 Ruaño G: Quo vadis personalized medicine. Personalized Med 2004;1:1-7.

15 Osborn LA: Psychiatry and Medicine: An Introduction to Personalized Medicine. New York, McGraw Hill, 1952.

16 de Leon J: The crucial role of the therapeutic window in understanding the clinical relevance of the poor versus the ultrarapid metabolizer phenotypes in subjects taking drugs metabolized by CYP2D6 and CYP2C19. J Clin Psychopharmacol 2007;27: 241-245.

17 Cappell K, Arndt M, Carey J: Drugs get smart. Business Week, 2005, pp 76-85.

18 de Leon J, Susce MT, Murray-Carmichael E: The AmpliChip CYP450 Genotyping Test: integrating a new clinical tool. Mol Diagn Ther 2006;10:135-151.
19 de Leon J: The AmpliChip CYP450 Test: personalized medicine has arrived in psychiatry. Expert Rev Mol Diagn 2006;6:277-286.

20 FDA: Guidance for industry: pharmacogenomic data submission. 2005. www.fda.gov/downloads/ RegulatoryInformation/Guidances/ucm 126957. pdf.

21 FDA: In Vitro Diagnostic Multivariate Index Assays (IVDMIAs). 2008.

22 FDA: Information for healthcare professionals: carbamazepine (marketed as Carbatrol, Equetro, Tegretol, and generics). 2007.

23 de Leon J, Armstrong SC, Cozza KL: Clinical guidelines for psychiatrists for the use of pharmacogenetic testing for CYP450 2D6 and CYP450 2C19. Psychosomatics 2006;47:75-85.

24 de Leon J, Arranz MJ, Ruaño G: Products for pharmacogenetic testing in psychiatry: a review of features and clinical realities. Clin Lab Med 2008;28: 599-617.

25 de Leon J, Susce MT, Pan RM, Fairchild M, Koch W, Wedlund PJ: The CYP2D6 poor metabolizer phenotype may be associated with risperidone adverse drug reactions and discontinuation. J Clin Psychiatry 2005;66:15-27.

26 de Leon J, Susce MT, Pan RM, Wedlund PJ, Orrego ML, Diaz FJ: A study of genetic (CYP2D6 and $\mathrm{ABCB} 1$ ) and environmental (drug inhibitors and inducers) variables that may influence plasma risperidone levels. Pharmacopsychiatry 2007;40:93102.

27 de Leon J, Armstrong SC, Cozza KL: A preliminary attempt to personalize risperidone dosing using drug-drug interactions and genetics. Part I. Psychosomatics 2008;49:258-270.

28 de Leon J, Armstrong SC, Cozza KL: A preliminary attempt to personalize risperidone dosing using drug-drug interactions and genetics. Part II. Psychosomatics 2008;49:347-361.

29 Lieberman JA, Stroup TS, McEvoy JP, Swartz MS, Rosenheck RA, Perkins DO, Keefe RSE, Davis SM, Davis CE, Lebowitz BD, Severe J, Hsaio JK: Effectiveness of antipsychotic drugs in patients with chronic schizophrenia. N Engl J Med 2005;353: 1209-1223.

Jose de Leon, MD

Mental Health Research Center at Eastern State Hospital

627 West Fourth St.

Lexington, KY 40508 (USA)

Tel. +1 859246 7563, Fax +1 859246 7019, E-Mail jdeleon@uky.edu 